\title{
A Comparative Study on Magnetostructural Properties of Barium Hexaferrite Powders Prepared by Polyethylene Glycol
}

\author{
Zehra Durmus \\ Department of Pharmaceutical Biotechnology, Bezmialem Vakıf University, Fatih, 34093 Istanbul, Turkey \\ Correspondence should be addressed to Zehra Durmus; zdurmus@bezmialem.edu.tr
}

Received 21 October 2014; Revised 9 December 2014; Accepted 17 December 2014; Published 31 December 2014

Academic Editor: Amir Kajbafvala

Copyright (C) 2014 Zehra Durmus. This is an open access article distributed under the Creative Commons Attribution License, which permits unrestricted use, distribution, and reproduction in any medium, provided the original work is properly cited.

\begin{abstract}
Nanocrystalline particles of barium hexaferrite were synthesized by a sol-gel combustion route using nitrate-citrate gels prepared from metal nitrates and citric acid solutions with $\mathrm{Fe} / \mathrm{Ba}$ molar ratio 12. The present paper aims to study the effect of addition of polyethylene glycol (PEG) solutions with different molecular weights (MW: 400, 2000, and 10.000 g/mol) on magnetostructural properties of barium hexaferrite. The formation of the barium hexaferrite was inspected using X-ray diffraction (XRD) analysis, Fourier transform infrared (FT-IR) analysis, thermogravimetric (TGA) analysis, scanning electron microscopy (SEM) analysis and vibrating sample magnetometer (VSM) analysis for magnetic measurements.
\end{abstract}

\section{Introduction}

Nanostructured magnetic materials have been intensively studied, due to their applications in magnetic high-density recording media, sensors, and biomolecular separations. Driven by radar electronics, wireless technologies, and enormous progress in fundamental theoretical and experimental laboratory studies of hexagonal $\mathrm{M}$ type ferrites various properties, researchers have shown huge interest in it. The best-known representative of the hexaferrite family, barium hexaferrite, has the uniaxial magnetoplumbite structure with a close-packed oxygen lattice forming $S R S^{*}{ }^{*}$ hexagonal blocks and a stoichiometry of $\mathrm{BaFe}_{12} \mathrm{O}_{19}(\mathrm{BaM})$. It is a high performance permanent magnetic material, well known by its fairly low cost, relatively high coercivity, excellent chemical stability, corrosion resistance, large magnetocrystalline anisotropy, and high Curie temperature [1]. As a result of their moderate coercivity, hexaferrite powders are suitable for magnetic-recording media applications. Moreover, due to their high anisotropy field, hexaferrites can be used at much higher frequencies than spinel ferrites or garnets. For this reason they are good candidates for applications above $30 \mathrm{GHz}$ based on particle size and sintering temperature $[2,3]$.

There are many processes for obtaining high quality magnetoplumbite powders such as hydrothermal [4], sol-gel method [5], aerosol pyrolysis technique [6], glass crystallization [7], microemulsion [8], self-propagation [9], and coprecipitation method [10]. Several techniques have been employed to obtain PEG modified magnetic nanoparticles using functional PEGs or engineered copolymers of PEG with functional groups such as $-\mathrm{OH}$ and $-\mathrm{COOH}$ that can interact with the free hydroxyls by hydrogen bonds surface of the particle [11]. The coprecipitation technique is suitable for the production of large quantities of magnetic nanoparticles but does not provide control over the nucleation and growth stages that govern the formation of the nanoparticles with a broad size distribution which are usually agglomerated, even after surface modification with surfactants or polymers. It has also been found that the morphology, agglomeration, and magnetization of nanoferrites particles can be controlled by selecting different types of PEG and different concentrations of PEG in the sol solutions. Han et al. investigated effects of adding different amounts of PEG2000 using the sol-gel synthesis technique and has concluded that ferrites prepared with more PEG added have lower coercivity and smaller $K_{1}$ values [11]. Prithviraj Swamy et al. prepared barium ferrite nanoparticles using metal oxalate precursors mixed with PEG by self-propagating low-temperature combustion route. They reported that, using polyethylene glycol (PEG) as a suitable economic fuel, good waterproof surfactant to form a shell 


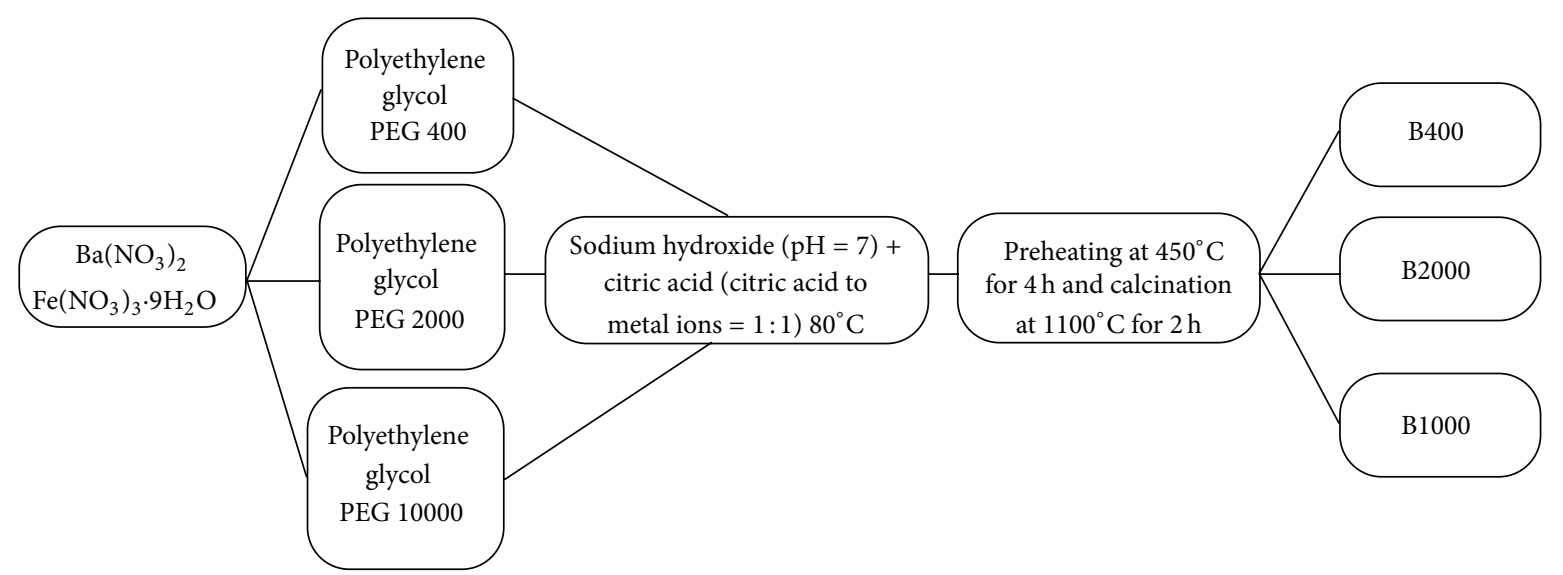

FIGURE 1: Schematic diagram of preparation the barium hexaferrite powders.

around the magnetic particles and a dispersant is perfect pathway for producing high-quality ferrite nanosized powder [12].

In this research, a sol-gel combustion technique using nitrate-citrate gels prepared from metal nitrates and citric acid solutions has been applied to synthesize barium hexaferrite nanopowder. It is a low-cost technique suitable for the mass production, when compared to the other mentioned methods, and the formation temperature of barium hexaferrite and its crystallite size in presence of polyethylene glycol with different molecular weight were compared [13]. Effect of chain length of polyethylene glycol is investigated on formation of its physical structure and magnetic properties.

\section{Experimental}

2.1. Synthesis. All the chemicals employed in the study were analytical grade and used as-received without a purification. Stoichiometric amounts of $\mathrm{Fe}\left(\mathrm{NO}_{3}\right)_{3} \cdot 9 \mathrm{H}_{2} \mathrm{O}$ and $\mathrm{Ba}\left(\mathrm{NO}_{3}\right)_{2}$ (Fe/Ba molar ratio of $12: 1$ ) were dissolved into three polyethylene glycol (PEG) solutions with MW: 400, 2000, and 10.000 , by vigorous stirring, and samples were named as B400, B2000, and B10000, respectively. Citric acid was added to the above solution as chelating agent under vigorous stirring with the molar ratios of citric acid to metal ions as $1: 1$ and $\mathrm{pH}$ was evaluated to 7 with ammonia at $50^{\circ} \mathrm{C}$. Finally PEG containing three different sols slowly evaporated at $80^{\circ} \mathrm{C}$ under constant stirring until a viscous gel was formed. By increasing the temperature up to $130^{\circ} \mathrm{C}$, the gel precursors were combusted to form brown loose powders with selfignition. Then obtained precursor powders were precalcined at $450^{\circ} \mathrm{C}$ for $4 \mathrm{~h}$ and sintered at $1100^{\circ} \mathrm{C}$ for $2 \mathrm{~h}$. $\mathrm{BaFe}_{12} \mathrm{O}_{19}$ nanopowders were thus obtained by citrate precursors using sol to gel (S-G) followed by gel to nanocrystalline (G-N) conversion and nomenclature of polyethylene glycol assisted barium hexaferrite nanopowders with self-ignition detailed in Figure 1. The formation of dendritic shape of BaM-rEGO nanocomposite was given before calcination in Figure 2.

2.2. Structural and Physical Characterization. The XRD patterns of the samples were obtained at room temperature by

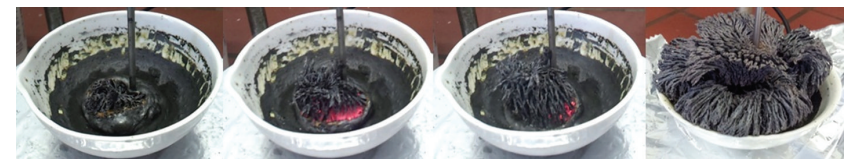

FIGURE 2: Photographs of formation of dendritic shape of polyethylene glycol assisted barium hexaferrite nanopowders with selfignition before calcination.

a Rigaku Smart Lab. XRD using $\mathrm{Cu}-\mathrm{K}_{\alpha}$ radiation. Fourier transform infrared (FT-IR) spectra of the samples were recorded with a Bruker Alpha infrared spectrometer in the range of $4000-400 \mathrm{~cm}^{-1}$. The surface morphology and microstructure of the samples were examined with a scanning electron microscope (JEOL 6335F, Field Emission Gun). Powder samples were directly imaged in the electron microscope after a proper sample preparation of sputter-coated with gold. Magnetic properties of the samples, all in powder form, were characterized with a vibrating sample magnetometer (VSM, LDJ Electronics Inc., Model 9600) at room temperature, in an applied field of $15 \mathrm{kOe}$. The thermal stability was determined by thermogravimetric analysis (TGA, Perkin Elmer Instruments model, STA 6000). The TGA thermograms were recorded for $5 \mathrm{mg}$ of powder sample at a heating rate of $10^{\circ} \mathrm{C} / \mathrm{min}$ in the temperature range of $30^{\circ} \mathrm{C}-800^{\circ} \mathrm{C}$ under nitrogen atmosphere.

\section{Results and Discussion}

3.1. FT-IR Analysis. Figure 3 shows the FT-IR spectra of precursor and calcined powders of B400, B2000, and B10.000 samples. All FTIR spectra indicate the characteristic absorption bands between 570 and $415 \mathrm{~cm}^{-1}$ corresponding to vibrations of the tetrahedral and octahedral sites for $\mathrm{BaFe}_{12} \mathrm{O}_{19}$ [14]. The band at $1398 \mathrm{~cm}^{-1}$ is attributed to the characteristic band of $\mathrm{NO}_{3}{ }^{-}$and $\mathrm{CO}_{2}{ }^{-}$in Figure 3(a) [15]. Also the samples of precursors of B2000 and B10000 show absorption band centered at $3010 \mathrm{~cm}^{-1}$ corresponding to hydrogen bonded $\mathrm{O}-\mathrm{H}$ stretching from absorbed water and the absorption bond due to the bending mode of $\mathrm{H}_{2} \mathrm{O}$ molecule around $1699 \mathrm{~cm}^{-1}$ is diagnostic of the presence of water of hydration 


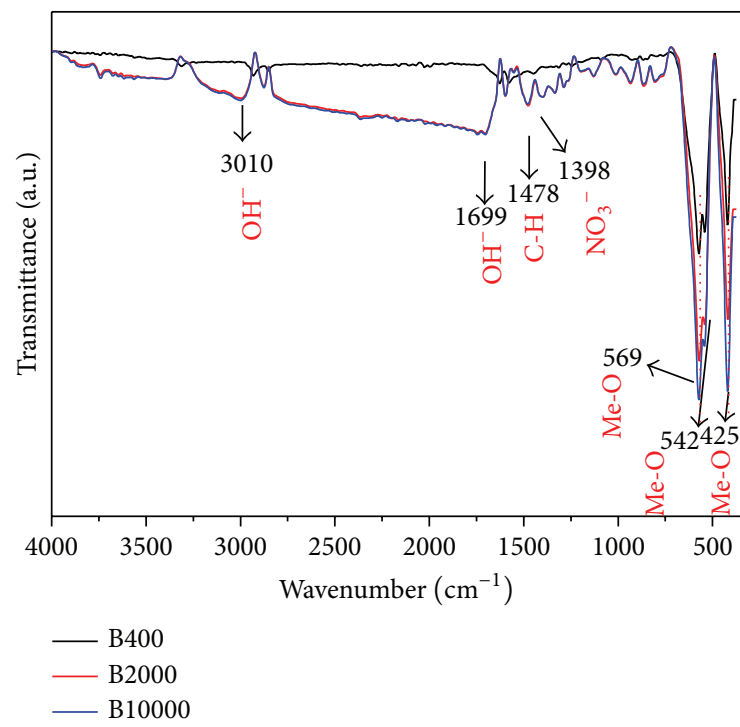

(a)

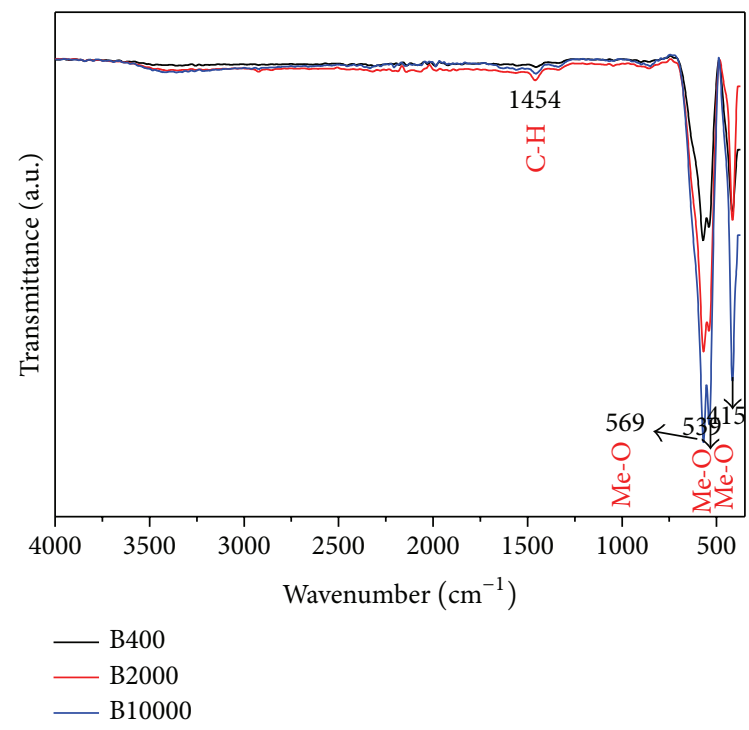

(b)

FIGURE 3: FTIR spectra of (a) precursor powders of B400, B2000, and B10.000 and (b) calcined powder of B400, B2000, and B10000 (annealing at $\left.1100^{\circ} \mathrm{C} \mathrm{Ba}^{2+} / \mathrm{Fe}^{3+}=11\right)$.

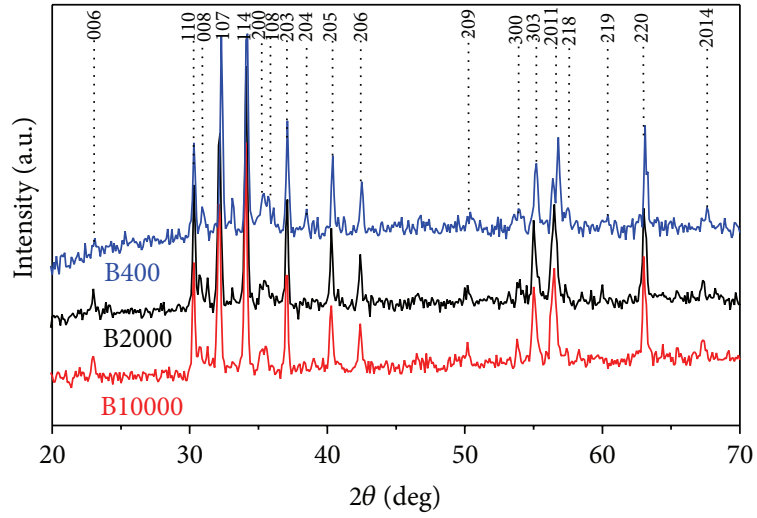

(a)

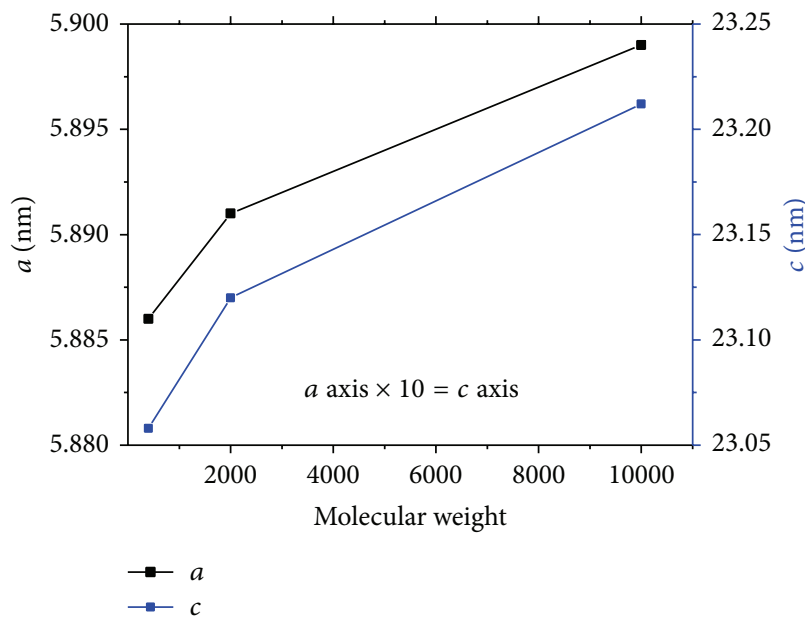

(b)

FIGURE 4: (a) XRD patterns and (b) lattice parameters of B400, B2000, and $\mathrm{B} 10.000$ (annealing at $1100^{\circ} \mathrm{C} \mathrm{Ba}^{2+} / \mathrm{Fe}^{3+}=11$ ) with respect to MW of PEG.

of both samples [16, 17]. The peak at $1478 \mathrm{~cm}^{-1}$ belongs to characteristic absorption peaks due to the vibration of $\mathrm{C}-$ $\mathrm{H}$ in PEG. FT-IR spectrum shows that the product does not exhibit any strong IR-active peak corresponding to impurities for all calcined samples except metal-oxygen bonds at $1100^{\circ} \mathrm{C}$ in Figure 3(b).

3.2. XRD Analysis. Figure 4(a) presents the X-ray diffractograms for calcined specimens of barium hexaferrite with prepared adding different molecular weight polyethylene glycols (PEG, MW: 400, 2000, and 10.000, which are named as B400, B2000, and B10000), respectively. The standard patterns for calcined hexagonal barium ferrite are also given in Figure 4 based on the JCPDS card 84-0757 clearly revealing that hexaferrites phase is formed after annealing at $1100^{\circ} \mathrm{C}$. From Figure 4(a), all the peaks can be indexed as M-type hexagonal structure with the following miller indices: (006), (110), (008), (107), (114), (200), (108), (203), (204), (205), (206), (209), (300), (303), (2011), (218), (219), (220), and (2014). The lattice parameters " $a$ and $c$ " were computed using the $d$-spacings and the respective (hkl) parameters and given in Figure 4(b).

3.3. SEM Analysis. Figure 5 shows the SEM micrographs of $\mathrm{BaFe}_{12} \mathrm{O}_{19}$ powders synthesized by adding different molecular weight polyethylene glycols by citrate melt method with self-ignition. The figure indicates the hexagonal plateletlike particles which dominate in the products are strongly 


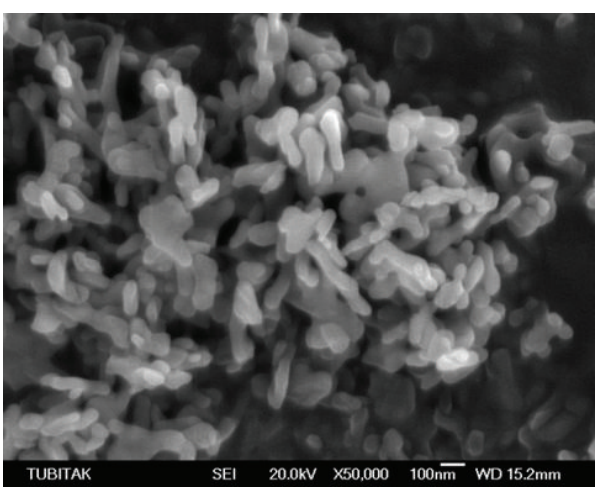

(a)

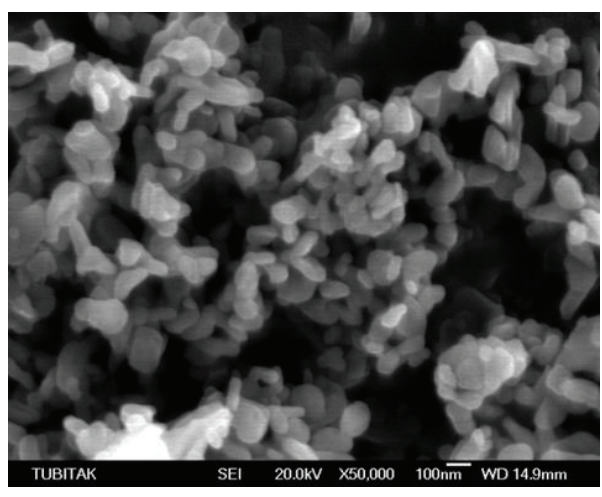

(c)

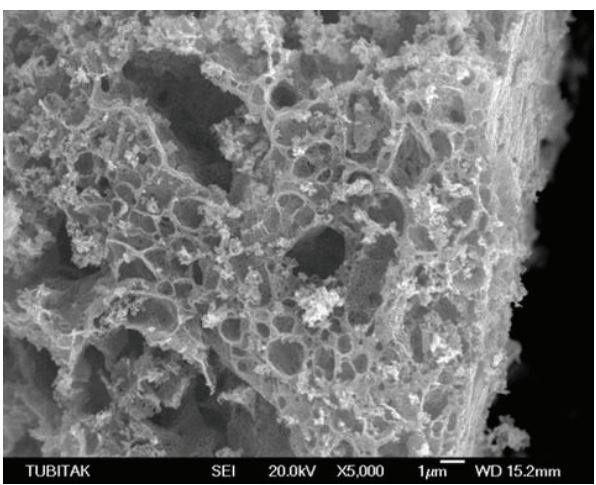

(e)

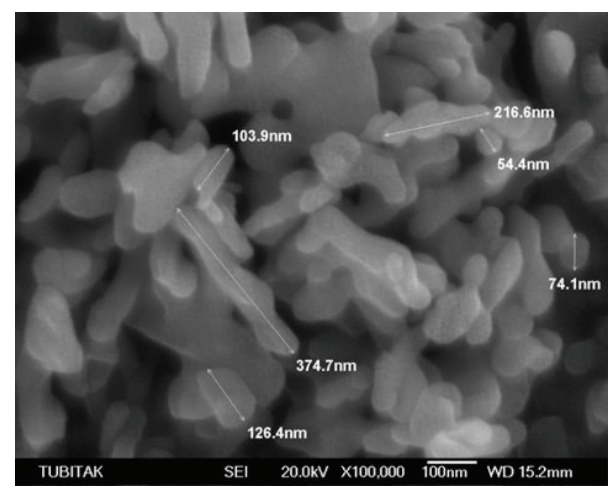

(b)

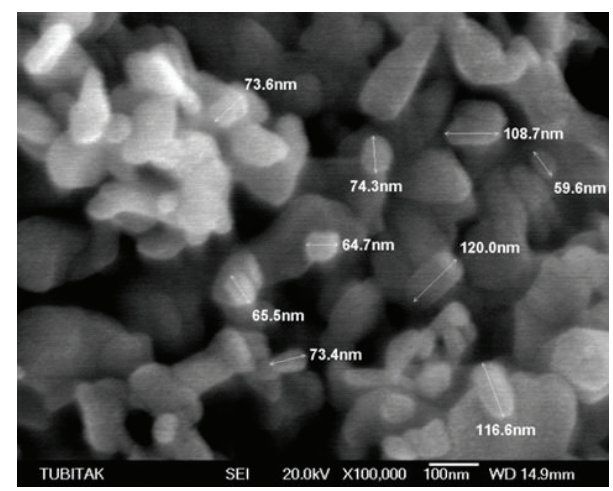

(d)

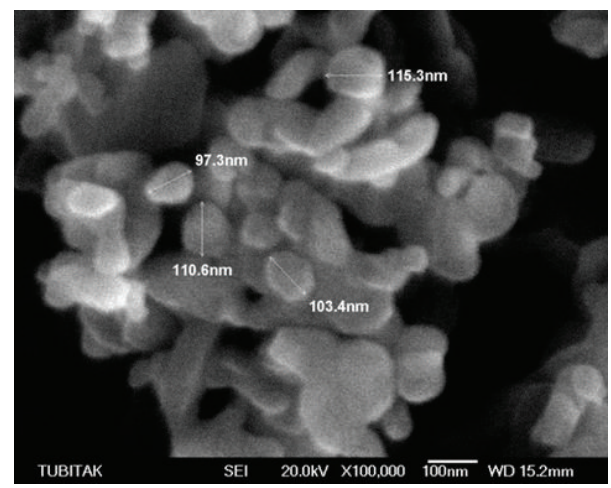

(f)

Figure 5: SEM micrographs of calcined samples (a, b) B400, (c, d) B2000, and (e, f) B10000 (annealing at $1100^{\circ} \mathrm{C} \mathrm{Ba}^{2+} / \mathrm{Fe}^{3+}=11$ ).

necked and relatively disorderly. The size of these particles varies between 50 and $400 \mathrm{~nm}$. This particle shape is typical observation for M-type hexaferrite nanoparticles obtained by hydrothermal process. In addition to this fact, selfpropagated burning samples, lacks of a template and fueled medium with different molecular weight polyethylene glycols, which do not allow the $\mathrm{BaFe}_{12} \mathrm{O}_{19}$ particles to grow as orientated. Some whole structures have been observed in all samples because of decomposed gases of long chained alcoholic structure of polyethylene glycol, these gases are released out from precursor samples which has been confirmed with FT-IR spectrums' of precursor samples.

3.4. TG Analysis. Figure 5 shows the TG patterns of B400, B2000, and B10.000 samples. For all samples, a two-step weight loss is observed from Figure 6. The initial weight loss is due to the loss of water in the precursors in the range of $20-200^{\circ} \mathrm{C}$. The second sharp weight loss had suddenly been observed around $210^{\circ} \mathrm{C}$ with transformation of mixed hydroxides into their oxides, which is attributed to thermal decomposition of the PEG gel chains.

The self-ignition could be considered as a thermally induced anionic, redox reaction of the gel wherein the citrate and nitrate ions behave as reductant and oxidant agent. Since the nitrate ions provide an in situ oxidizing medium for the decomposition of the citrate, the rate of oxidation reaction increases slightly. The combination of the lowering of the reaction temperature and the increase in rate results in a self-propagating combustion of the nitrate-citrate gel $[18,19]$. So the first sharp exothermic peak could be due to an 


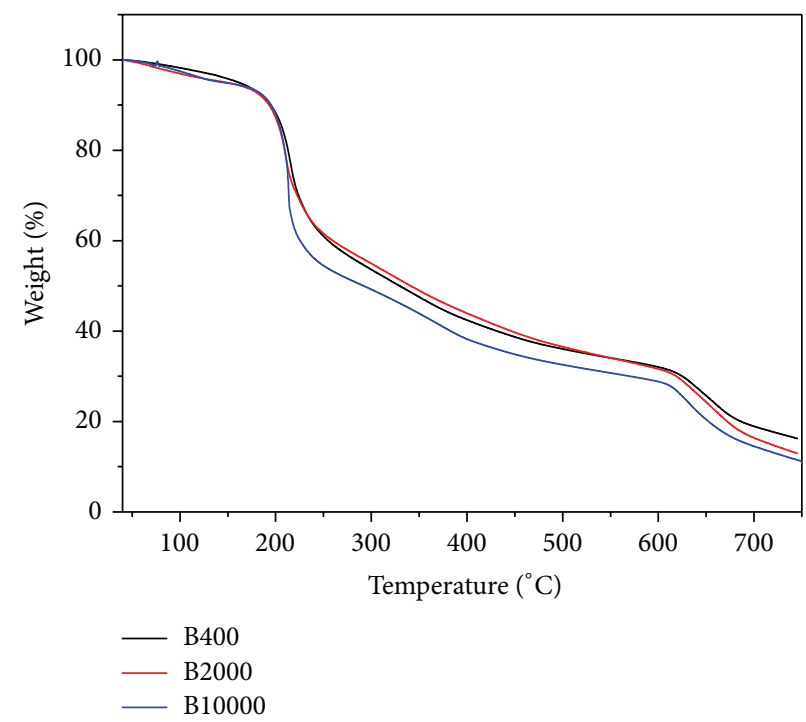

FIGURE 6: TGA thermograms of precursor powder of B400, B2000, and $\mathrm{B} 10000$ samples (annealing at $1100^{\circ} \mathrm{C} \mathrm{Ba}^{2+} / \mathrm{Fe}^{3+}=11$ ).

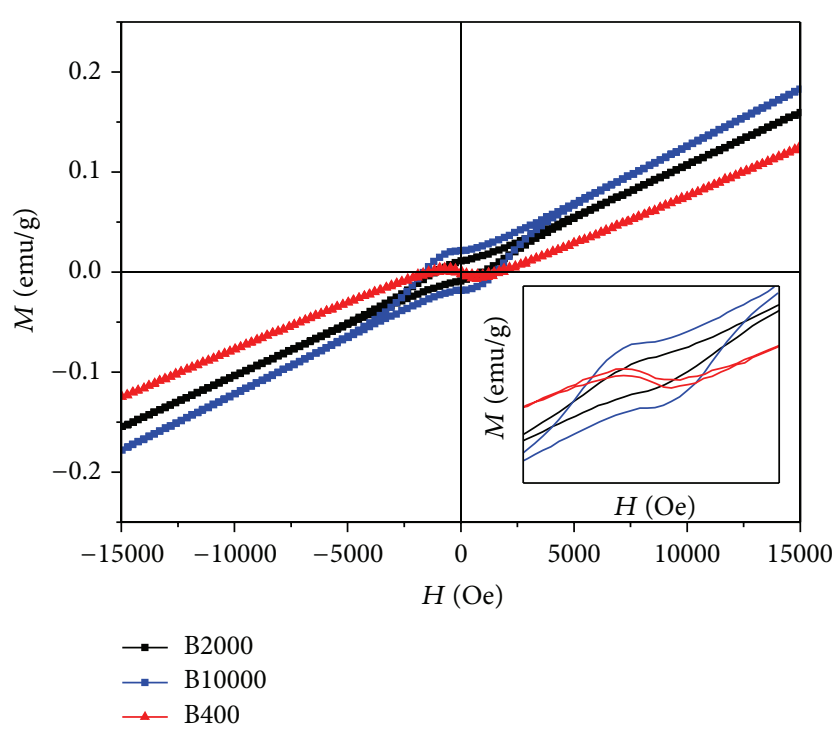

(a)

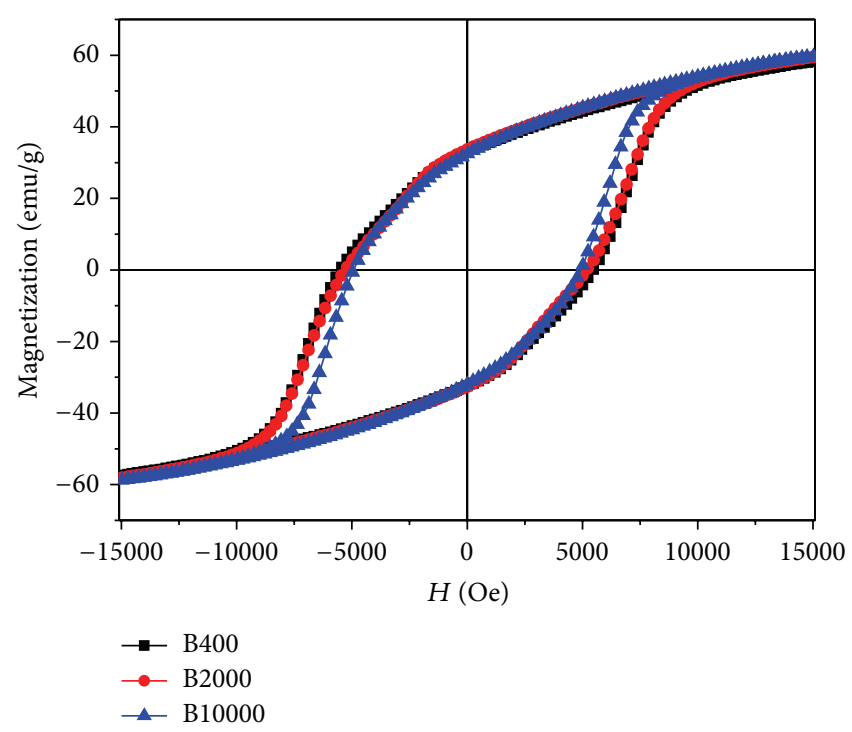

(b)

Figure 7: $M-H$ hysteresis curve of (a) B400 and B2000 and B10.000 precursor and (b) calcined powders of B400, B2000, and B10.000 (annealing at $1100^{\circ} \mathrm{C} \mathrm{Ba}^{2+} / \mathrm{Fe}^{3+}=11$ ) at room temperature.

autocatalytic anionic oxidation-reduction reaction between the nitrates and citric acid. However, the decomposition of unreacted citric acid that remained after combustion could be responsible for the second exothermic peak at about $610^{\circ} \mathrm{C}$ with a weight loss of $14 \%$. During the combustion, large amounts of gases such as $\mathrm{H}_{2} \mathrm{O}, \mathrm{CO}, \mathrm{CO}_{2}$, and $\mathrm{NO}$ are released. The weight losses reach to $84.3 \%, 88.2 \%$, and $90 \%$ for $\mathrm{B} 400$, B2000, and B10000 in the end of second step, respectively.

3.5. VSM Analysis. Figure 7(a) shows the plots of the saturation magnetization $\left(M_{s}\right)$ as a function of the applied field $\left(H_{c}\right)$ in the field range of $\pm 15 \mathrm{kOe}$ for samples prepared by polyethylene glycol annealing at $1100^{\circ} \mathrm{C} \mathrm{Ba}{ }^{2+} / \mathrm{Fe}^{3+}=11$ at room temperature using a vibrating sample magnetometer.
The precursors obtained during the self-ignition step at 130 $150^{\circ} \mathrm{C}$ show the paramagnetic nature of metal oxides with diamagnetic effects at low fields.

In Figure 7(b) it is shown that the pure barium hexaferrite nanoparticles are synthesized with PEG (molecular weight, MW: 400, 2000, and $10.000 \mathrm{~g} / \mathrm{mol}$ ). All samples do not show the saturated magnetization despite high magnetic field. The saturation magnetization values are estimated from the extrapolating of $M$ versus $1 / H$ curves when $1 / H$ goes to zero. The obtained results are $71.15,72.18$, and $71.63 \mathrm{emu} / \mathrm{g}$ for hexaferrite with MW of PEG 400, 2000, and 10000, respectively. The remanent magnetization values are 33,65 and 32,44 with increasing MW of PEG while $M_{s}$ and $M_{r}$ do not vary with different MW of coating PEG. It is to be noted 
that the remarkable coercive field change is observed. The $H_{c}$ values are 6460,5190 , and 4935 Oe with increasing MW of PEG by using high MW of PEG. The degrees of magnetism of each calcined sample were also close to results of work by Baykal et al. [20].

\section{Conclusion}

In the present study three barium hexaferrite nanoparticles were prepared with the addition of PEG with different molecular weight. The final product was analyzed for composition, microstructure, thermal behavior, and magnetization. Samples have been observed with high specific saturation magnetization and coercivity, which is synthesized via a simple citrate-nitrate combustion using sol to gel (S-G) followed by a gel to nano crystalline (G-N) conversion route, while precalcinated sample had not showed the feature of specific saturation magnetization and exhibited negligible coercivity. Morphological structure of barium hexaferrite phase was confirmed by XRD and SEM analysis. These particles show nearly single crystalline nature. The interaction between PEG and $\mathrm{BaFe}_{12} \mathrm{O}_{19}$ nanoparticles was confirmed by FT-IR spectroscopy for precursor samples. Magnetic measurements have shown that while precursor samples are formed as paramagnetic (very small ferromagnetic) material, calcined strontium ferrite powders have saturation magnetization $\left(M_{s}\right)$ values $71.15,72.18$, and $71.63 \mathrm{emu} / \mathrm{g}$ for MW of PEG 400, 2000 , and 10000 at the annealed temperature at $1100^{\circ} \mathrm{C}$ for $2 \mathrm{~h}$ with $\mathrm{Fe} / \mathrm{Sr}$ ratio of 11.

\section{Conflict of Interests}

The author declares that there is no conflict of interests regarding the publication of this paper.

\section{Acknowledgment}

The author is thankful to the Bezmialem Vakif University Research Project Foundation (Project no.: 9.2013/4) for financial support of this study.

\section{References}

[1] W. K. Ng, J. Ding, Y. Y. Chow, S. Wang, and Y. Shi, "A study on barium ferrite particles prepared by chemical coprecipitation," Journal of Materials Research, vol. 15, no. 10, pp. 2151-2156, 2000.

[2] S. B. Thompson and G. P. Rodrigue, "The application of planar anisotropy to millimeter-wave ferrite Phase Shifters," IEEE Transactions on Microwave Theory and Techniques, vol. 33, no. 11, pp. 1204-1209, 1985.

[3] D. Lisjak and M. Drofenik, "The mechanism of the low-temperature formation of barium hexaferrite," Journal of the European Ceramic Society, vol. 27, no. 16, pp. 4515-4520, 2007.

[4] D. Barb, L. Diamandescu, A. Rusi, D. Tărăbăsanu-Mihăilă, M. Morariu, and V. Teodorescu, "Preparation of barium hexaferrite by a hydrothermal method: structure and magnetic properties," Journal of Materials Science, vol. 21, no. 4, pp. 1118-1122, 1986.
[5] W. Zhong, W. Ding, N. Zhang, J. Hong, Q. Yan, and Y. Du, "Key step in synthesis of ultrafine $\mathrm{BaFe}_{12} \mathrm{O}_{19}$ by sol-gel technique," Journal of Magnetism and Magnetic Materials, vol. 168, no. 1-2, pp. 196-202, 1997.

[6] Z. X. Tang, S. Nafis, C. M. Sorensen, G. C. Hadjipanayis, and K. J. Klabunde, "Magnetic properties of aerosol synthesized barium ferrite particles," IEEE Transactions on Magnetics, vol. 25, no. 5, pp. 4236-4238, 1989.

[7] L. Rezlescu, E. Rezlescu, P. D. Popa, and N. Rezlescu, "Fine barium hexaferrite powder prepared by the crystallisation of glass," Journal of Magnetism and Magnetic Materials, vol. 193, no. 1-3, pp. 288-290, 1999.

[8] X. Liu, J. Wang, L.-M. Gan, S.-C. Ng, and J. Ding, "An ultrafine barium ferrite powder of high coercivity from water-in-oil microemulsion," Journal of Magnetism and Magnetic Materials, vol. 184, no. 3, pp. 344-354, 1998.

[9] I. P. Parkin, G. Elwin, L. F. Barquín et al., "Self-propagating high temperature synthesis of hexagonal ferrites $\mathrm{MFe}_{12} \mathrm{O}_{19}(\mathrm{M}=\mathrm{Sr}$, Ba)," Advanced Materials, vol. 9, no. 8, pp. 643-645, 1997.

[10] S. R. Janasi, D. Rodrigues, F. J. G. Landgraf, and M. Emura, "Magnetic properties of coprecipitated barium ferrite powders as a function of synthesis conditions," IEEE Transactions on Magnetics, vol. 36, no. 5, pp. 3327-3329, 2000.

[11] M. G. Han, Y. Ou, W. Chen, and L. Deng, "Magnetic properties of Ba-M-type hexagonal ferrites prepared by the sol-gel method with and without polyethylene glycol added," Journal of Alloys and Compounds, vol. 474, no. 1-2, pp. 185-189, 2009.

[12] P. M. Prithviraj Swamy, S. Basavaraja, V. Havanoor, N. V. Srinivas Rao, R. Nijagunappa, and A. Venkataraman, "Barium ferrite nanoparticles prepared by self-propagating low-temperature combustion method and its characterization," Bulletin of Materials Science, vol. 34, no. 7, pp. 1319-1323, 2011.

[13] M. G. Hasab, S. A. S. Ebrahimi, and A. Badiei, "Effect of different fuels on the strontium hexaferrite nanopowder synthesized by a surfactant-assisted sol-gel auto-combustion method," Journal of Non-Crystalline Solids, vol. 353, no. 8-10, pp. 814-816, 2007.

[14] S. Singhal, T. Namgyal, J. Singh, K. Chandra, and S. Bansal, "A comparative study on the magnetic properties of $\mathrm{MFe}_{12} \mathrm{O}_{19}$ and $\mathrm{MAlFe}_{11} \mathrm{O}_{19}(\mathrm{M}=\mathrm{Sr}, \mathrm{Ba}$ and $\mathrm{Pb})$ hexaferrites with different morphologies," Ceramics International, vol. 37, no. 6, pp. 1833$1837,2011$.

[15] N. Koga and T. Tsutaoka, "Preparation of substituted barium ferrite $\mathrm{BaFe}_{12-x}\left(\mathrm{Ti}_{0.5} \mathrm{Co}_{0.5}\right)_{x} \mathrm{O}_{19}$ by citrate precursor method and compositional dependence of their magnetic properties," Journal of Magnetism and Magnetic Materials, vol. 313, no. 1, pp. 168-175, 2007.

[16] C. Sudakar, G. N. Subbanna, and T. R. N. Kutty, "Nanoparticles of barium hexaferrite by gel to crystallite conversion and their magnetic properties," Journal of Electroceramics, vol. 6, no. 2, pp. 123-134, 2001.

[17] F. Khademi, A. Poorbafrani, P. Kameli, and H. Salamati, "Structural, magnetic and microwave properties of Eu-doped barium hexaferrite powders," Journal of Superconductivity and Novel Magnetism, vol. 25, no. 2, pp. 525-531, 2012.

[18] D.-X. Zhao, Q.-L. Li, Y. Ye, and C.-R. Zhang, "Synthesis and characterization of carbon nanotubes decorated with strontium ferrite nanoparticles," Synthetic Metals, vol. 160, no. 9-10, pp. 866-870, 2010. 
[19] A. Mali and A. Ataie, "Structural characterization of nanocrystalline $\mathrm{BaFe}_{12} \mathrm{O}_{19}$ powders synthesized by sol-gel combustion route," Scripta Materialia, vol. 53, no. 9, pp. 1065-1070, 2005.

[20] A. Baykal, Z. Durmus, M. S. Toprak, and H. Sozeri, "Synthesis and characterization of PEG-Sr hexaferrite by sol-gel conversion," Journal of Superconductivity and Novel Magnetism, vol. 25, no. 6, pp. 2003-2008, 2012. 

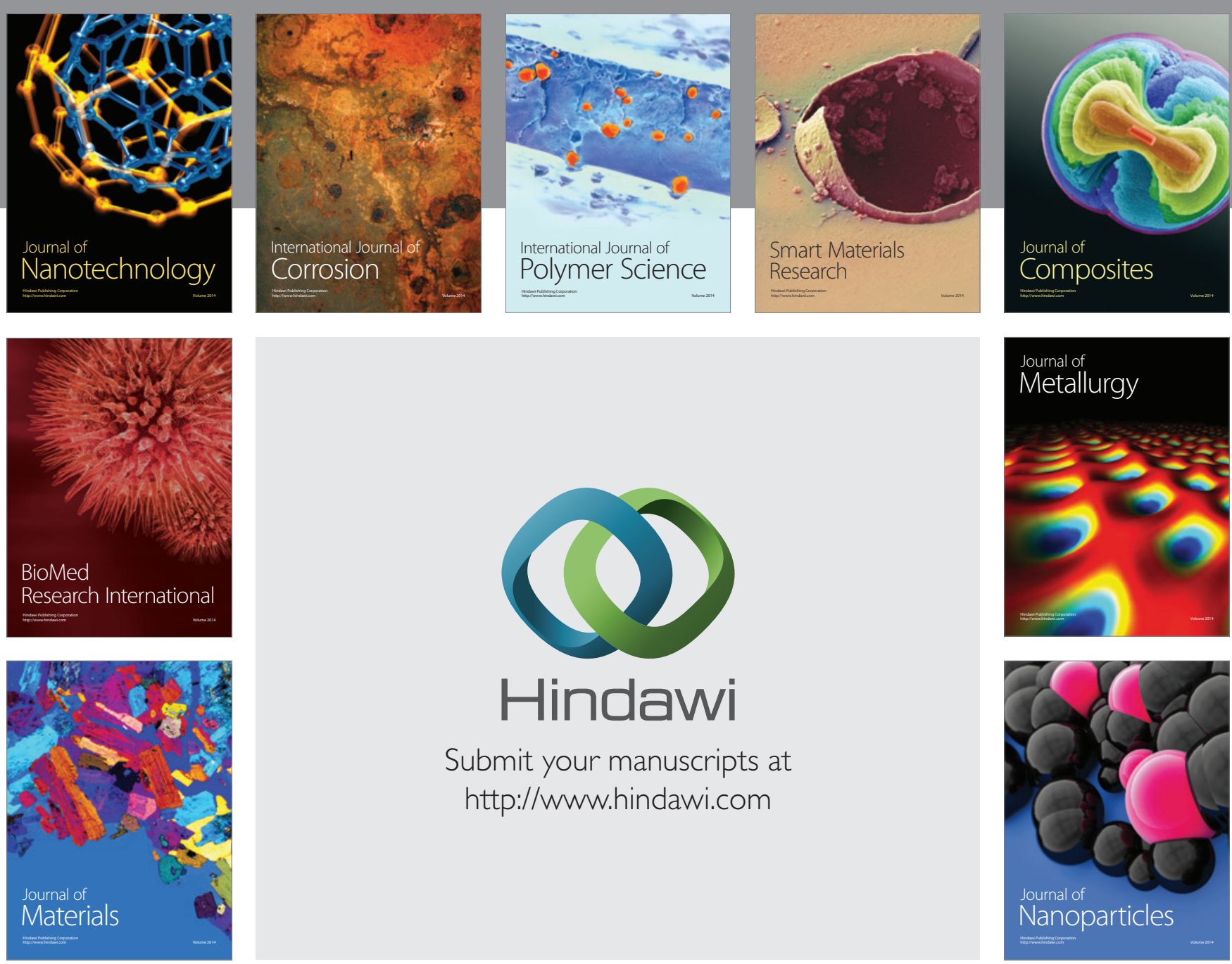

Submit your manuscripts at http://www.hindawi.com
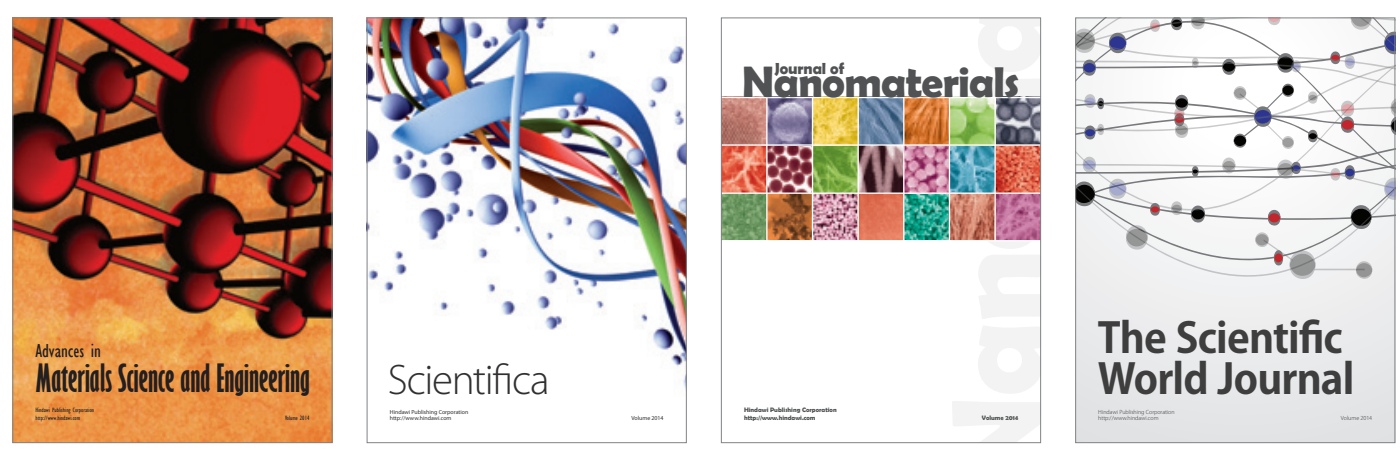

\section{The Scientific World Journal}
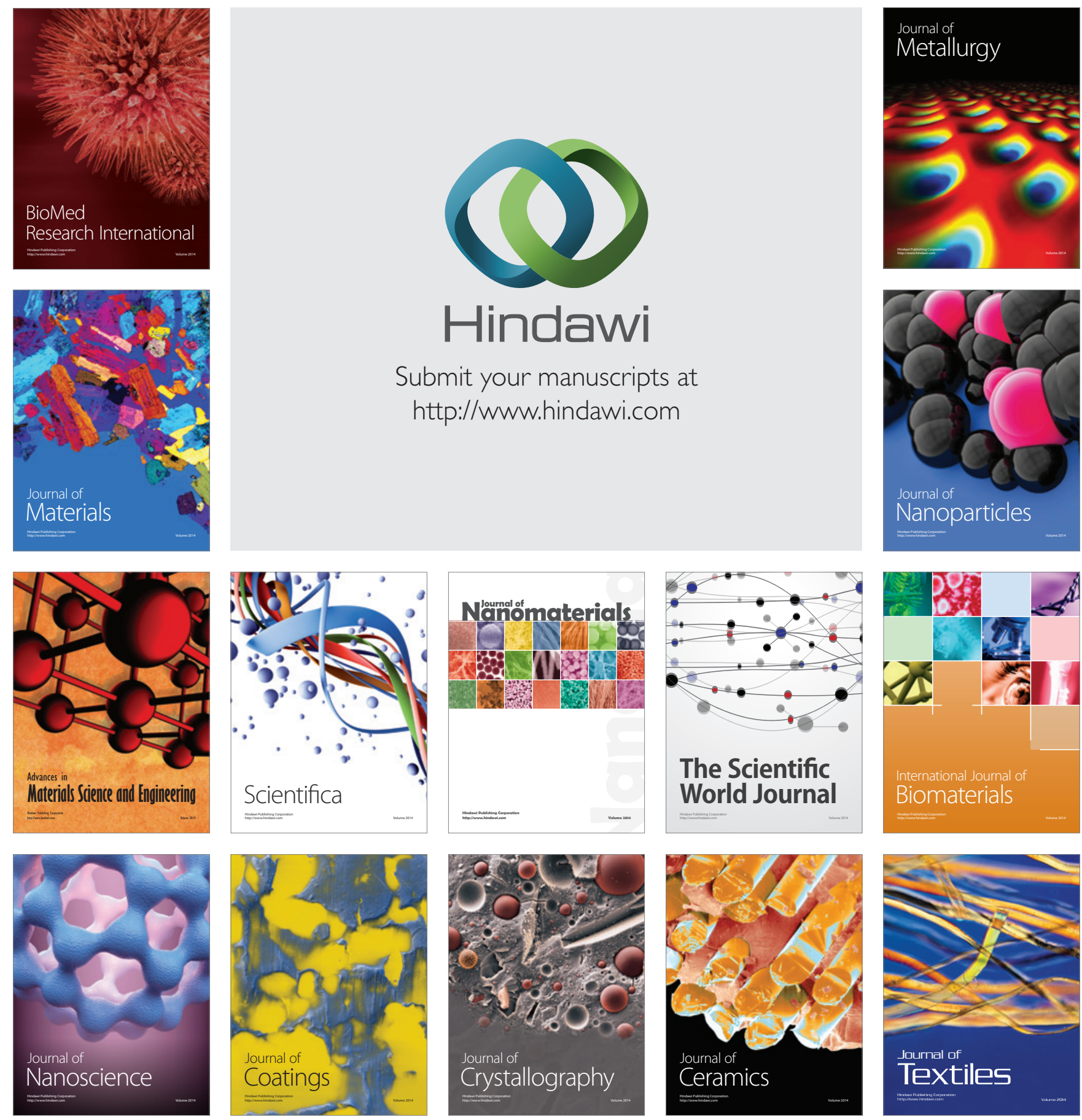